\title{
A Symbolic Method for Distortion Analysis and Optimization
}

\author{
J. Chen, G. Shi, A. Zhang \\ Department of Micro/Nano-Electronics \\ Shanghai Jiao Tong University \\ Shanghai, China
}

\begin{abstract}
A symbolic method is presented for calculation of the weakly nonlinear distortion effect of operational amplifiers. To avoid complicated symbolic generation by considering all transistor nonlinearities, a simplified analysis method is adopted. This method identifies the nonlinearity of each amplifier stage by a low-order polynomial. The proposed symbolic method can derive analytical distortion results automatically without the need of going through lengthy signal-flow graph analysis. This method is applied to sweep analysis of the opamp distortion with respect to the external feedback elements, by which optimal selection of the parameter value can be determined at any given frequency. Remarkable speedup over repeated Spectre simulation has been observed.
\end{abstract}

Keywords-distortion analysis; operational amplifier (opamp); optimization; symbolic analysis

\section{INTRODUCTION}

Advanced analog integrated circuits (IC) require high linearity for precision signal processing. However, along with severe nonlinearity, process variation is worsening as semiconductor manufacturing technology advances. In some cases the designers also need to optimize the distortion effect over a selected working frequency band. Both problems require proper characterization of distortion with respect to variational circuit parameters. A symbolic method can help in this regard.

In this work we present a symbolic distortion analysis method that can quickly generate analytical distortion results for an operational amplifier (opamp). There is already one symbolic distortion analysis method proposed in the literature by Wambacq et al. [1] a decade ago. However, since this method deals with all transistor nonlinearity analytically, the symbolic generation cost is extremely high and the design insights would be buried in overwhelming symbolic results if not simplified properly. Recently, a new technique has been adopted by several publications, which uses a numerical identification method for distortion characterization [2, 3, 4]. By this method each stage of an amplifier is modeled by a third-order polynomial two-port; the polynomial coefficients are identified by applying lowfrequency periodic excitation to each stage.

We review briefly the Volterra series theory for symbolic analysis in section 2, then simply describe the nonlinearity identification method in section 3 . The symbolic distortion analysis method is introduced in section 4. An application example is reported in section 5. Section 6 concludes the paper.

\section{REVIEW OF THE VOLTERRA SERIES METHOD}

Typically, opamps are designed in stages, such as one, two, three, or more stages. Each stage can be treated as two-port with one input port and one output port. The nonlinearity of each stage could contribute distortion to the whole circuit. In the works [2, 3, 4] a method based on identifying stage-wise nonlinearity seems to work quite well. A basic message is that it might be unnecessary to consider the detailed nonlinearities with each transistor for the circuit-level distortion characterization. As in [2, 3, 4], we assume that the nonlinearity of each stage takes a bivariate third-order polynomial function in its most general form.

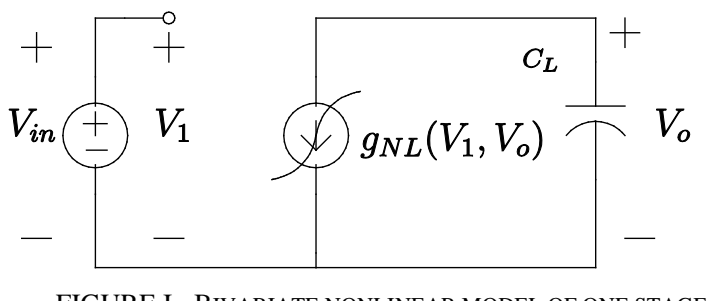

Shown in fig.1 is a nonlinear two-port circuit. The nonlinear element $g_{N L}\left(V_{1}, V_{o}\right)$ is assumed a polynomial function of $V_{1}$ (the input voltage) and $V_{o}$ (the output voltage). Many opamps have compensation elements connected between stages to improve stability; some of them are capacitive and can significantly affect distortion at specific frequency ranges. This is the main reason why many of the recent works have paid particular attention to high-frequency distortion analysis [2, 3, 4]. However, these works without exception used the traditional signal-flow method for deriving distortion formulas. Since high-order terms are involved in the derivation, although explicit formulas can be derived, their real usefulness is questionable because in general very little design insights can be appreciated. A more serious drawback is that whenever the circuit topology is changed even slightly, the derivation has to be conducted again.

This work is motivated by the limitation of the signal-flow method already used in the literature, and proposes to take the approach of symbolic analysis and incorporate the state-space analysis method. Due to the fact that each opamp stage is modelled by a low-order polynomial, symbolic analysis based on the Volterra series theory has very low complexity and can be automated. 
Note that in Volterra series analysis the first-order system is driven by the input source $V_{i n}=V_{0} e^{j \omega}$ while the rest two systems (up to the third-order)are respectively driven by virtual currents generated by the second- and third-order nonlinear terms of $g_{N L}\left(V_{1}, V_{o}\right)[1,4]$.

Apparently, the nodal voltages solved by a lower order system are used by the next order system. In practice, the sequence of equations is solved iteratively and a symbolic solver can speed up the process.

A bivariate nonlinearity is described by the following third order polynomial

$$
\begin{aligned}
i=g_{N L}\left(V_{1}, V_{o}\right) & \approx\left(g_{m 1} V_{1}+g_{o 1} V_{o}\right)+\left(g_{m 2} V_{1}^{2}+g_{m 101} V_{1} V_{o}+g_{o 2} V_{o}^{2}\right) \\
& +\left(g_{m 3} V_{1}^{3}+g_{m 201} V_{1}^{2} V_{o}+g_{m 102} V_{1} V_{o}^{2}+g_{o 3} V_{o}^{3}\right)
\end{aligned}
$$

The coefficients can be determined by harmonic probing of a circuit stage, which is to be discussed in Section 3. Denote by $h_{i, k}(j \omega)$ for the k-th order Volterra transfer function at the i-th node. It can be shown that the second- and third-order virtual currents are given by

$$
\begin{gathered}
I_{2,2}^{b i}=\left\{g_{m 2} h_{1,1}^{2}+g_{o 2} h_{o, 1}^{2}+g_{m 101} h_{1,1} h_{o, 1}\right\}_{(j \omega)} V_{i n}^{2} \\
I_{2,3}^{b i}=\left\{\begin{array}{l}
g_{m 3} h_{1,1}^{3}+g_{o 3} h_{o, 1}^{3}+g_{m 201} h_{1,1}^{2} h_{o 1}+g_{m 102} h_{11} h_{o 1}^{2}+ \\
\left.2 g_{m 2} h_{1,1} h_{1,2}+2 g_{o 2} h_{o, 1} h_{o, 2}+g_{m 101} h_{1,1} h_{o, 2}+h_{1,2} h_{o, 1}\right)
\end{array}\right\} V_{(j \omega)}^{3}
\end{gathered}
$$

where the subscripts( $j \omega)$ indicate at which frequency point the transfer functions are evaluated. The second- and third-order harmonic distortion factors of the nonlinear two-port are defined by [6].

$$
H D_{2}(j \omega)=\frac{V_{0}}{2}\left|\frac{h_{o, 2}(j \omega)}{h_{o, 1}(j \omega)}\right| H D_{3}(j \omega)=\frac{V_{0}^{2}}{4}\left|\frac{h_{o, 3}(j \omega)}{h_{o, 1}(j \omega)}\right|
$$

\section{NONLINEARITY IDENTIFICATION}

The works $[2,3,4]$ adopted the nonlinearity identification method, but none of them described the details. For completeness, we outline the detailed steps for harmonic probing. This method is numerically robust and fits nonlinear circuits the best. Other methods like using high order finite difference are not numerically stable. A nonlinear circuit inherently modulates a harmonic signal excitation. We may measure the harmonics by direct measurement or simulation [7]. To avoid exciting capacitive parasitics and high-order nonlinearity, we should apply a low-frequency and low-magnitude signal for probing. The coefficients are determined by matching theoretically derived coefficients to the numerically calculated harmonic components.

For example, consider the bivariate nonlinearity (1).Assuming $u=A \cos (\omega t), A=1$ and $v=0$, we get by trigonometric identities,

$$
\begin{aligned}
g_{N L}(u, 0) & =\frac{g_{m 1} A^{2}}{2}+\left(g_{m 1} A+\frac{3 g_{m 3} A^{3}}{4}\right) \cos (\omega t) \\
& +\frac{g_{m 2} A^{2}}{2} \cos (2 \omega t)+\frac{g_{m 3} A^{3}}{4} \cos (3 \omega t)
\end{aligned}
$$

Since the input magnitude $A$ is known, the numerically simulated/measured harmonic magnitudes can be used to calculate $g_{m 1}$ (at dc), $g_{m 2}$ (at $2 \omega$ ), and the component $g_{m 3}$ (at $\omega$ or $3 \omega)$. The other three coefficients $g_{o 1}, g_{o 2}$ and $g_{o 3}$ can be determined analogously by flipping the roles of $u$ and $v$. The coefficients of the cross-terms, i.e., $g_{m 101}, g_{m 201}$ and $g_{m 102}$, also can be determined by applying the probing voltages at both ports and measuring the current of the nonlinear element. That is, assuming $u=A \cos \left(\omega_{1} t\right)$ and $v=B \cos \left(\omega_{2} t\right)$ with $\omega_{1} \neq \omega_{2}$, we get

$$
g_{N L}(u, v)=G_{1}\left(w_{1}\right)+G_{2}\left(w_{2}\right)+g_{m 101} K_{1,1}+g_{m 201} K_{2,1} g_{m 102} K_{1,2}
$$

where $G_{1}\left(\omega_{1}\right):=g_{N L}(u, 0)$ and $G_{2}\left(\omega_{2}\right):=g_{N L}(0, v)$, and

$$
\begin{aligned}
& K_{1,1}:=\frac{1}{2} A B\left[\cos \left(\omega_{1}+\omega_{2}\right) t+\cos \left(\omega_{1}-\omega_{2}\right) t\right] \\
& K_{2,1}:=\frac{1}{4} A^{2} B\left[2 \cos \left(\omega_{2} t\right)+\cos \left(2 \omega_{1}+\omega_{2}\right) t+\cos \left(2 \omega_{1}-\omega_{2}\right) t\right] \\
& K_{2,1}:=\frac{1}{4} A B^{2}\left[2 \cos \left(\omega_{1} t\right)+\cos \left(\omega_{1}+2 \omega_{2}\right) t+\cos \left(\omega_{1}-2 \omega_{2}\right) t\right]
\end{aligned}
$$

By reading the harmonic component magnitudes (obtained by Fourier transform), we can calculate the cross-term coefficients.

\section{SYMBOLIC ANALYSIS METHOD}

Recall that different orders of distortion responses can be solved from the same linearized circuit driven by sources applied at appropriate places. A symbolic method is capable of solving a linear circuit with specific input and output (I/O). Moreover, a recently developed symbolic method has the feature of solving a linear circuit with multiport I/O by using a shared binary decision diagram (BDD) [8]. Another particular reason that we choose this method is that circuit parameters are uniquely coded as the symbols in the symbolic result, which is very suitable for sweeping and sensitivity analysis.

Shown in fig. 2 is a symbolic BDD representing the following two transfer functions

$$
H_{1}(s)=\frac{g_{m 1}}{g_{o 1}+s C}, \quad H_{2}(s)=\frac{1}{g_{o 1}+s C}
$$




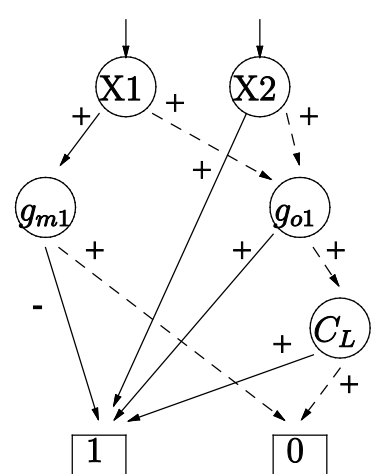

FIGURE II. ILLUSTRATION OF A TWO-ROOT SYMBOLIC BDD

The two roots at the top provide the access points to the two functions. Noting that the three MNA matrices corresponding to different order circuit in [1] differ only by a frequency multiplicity, a shared BDD with multiple roots (mapped from the $\mathrm{I} / \mathrm{O}$ relations) is an efficient data structure for saving the symbolic distortion coefficients.

\section{APPLICATION EXAMPLE}

Symbolically generated distortion results are saved in a BDD data structure. In most applications such symbolic results are used as a computation engine for post-processing, including generating numerical results. Because there is no need of repeated construction, numerical evaluation in most cases is much faster than repeatedly running circuit simulations. We implemented a C++ program for symbolic distortion analysis. This program can be used to perform distortion analysis of most opamps designed in stages. We use an opamp as an example to assess the accuracy of symbolic analysis and show the potential in optimizing distortion performance.

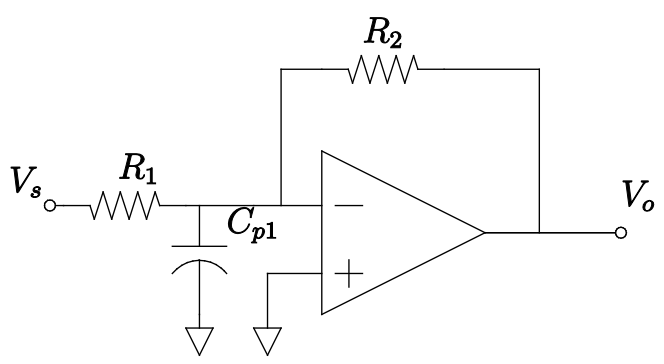

FIGURE III. CONNECTION OF OPAMP IN FEEDBACK

We assume the amplifier is connected as shown in fig. 3 which has an analog dc gain defined by $R_{2} / R_{1}$. The resistors $R_{1}$ and $R_{2}$ are turnable for shaping the distortion at different frequency ranges $[2,3,4]$. The capacitor $C_{p 1}$ represents the parasitics at the opamp input. In the experiment all transistor level circuits were simulated by the Cadence Spectre simulator using the TSMC 0.18um technology library.

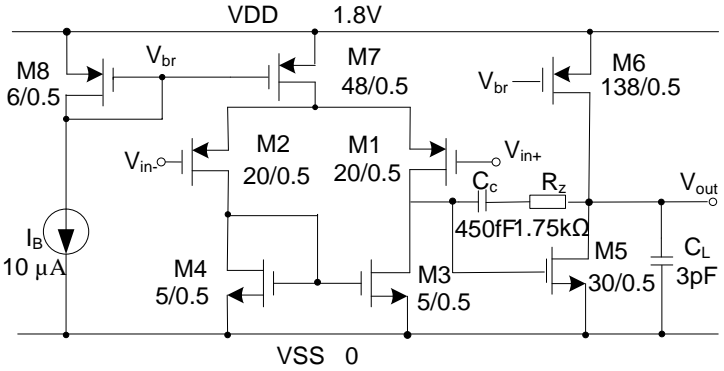

FIGURE IV. TWO-STAGE MiLLER-COMPENSATED OPAMP (PMOS-INPUT)

In the example we consider the two-stage Miller compensated opamp (with PMOS input pair) shown in fig.4. This amplifier has been sized to achieve dc gain $A_{d c}=73.9 \mathrm{~dB}$, $f_{3 d B}=35.9 \mathrm{KHz}$, and GBW $=178 \mathrm{MHz}$. Shown in fig. 5 is the corresponding nonlinear small-signal model, where $\mathrm{R}_{1}=\mathrm{R}_{2}=$ $10 \mathrm{k} \Omega, \mathrm{C}_{\mathrm{p} 1}=62 \mathrm{fF}$, and $\mathrm{C}_{\mathrm{p} 2}=86 \mathrm{fF}$.

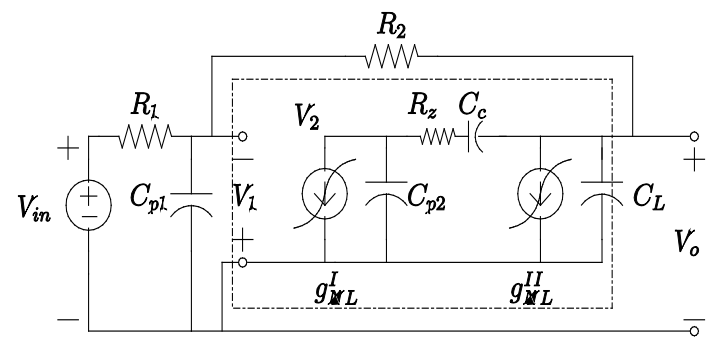

FIGURE V. NONLINEAR SMALL-SIGNAL MODEL FOR THE TWO-STAGE AMPLIFIER

TABLE I. IDENTIFIED NONLINEAR COEFFICIENTS FOR THE TWO-STAGE AMPLIFIER

\begin{tabular}{|c|c|c|c|c|c|}
\hline \multicolumn{2}{|c|}{$1^{\text {st }}$ order $(\mathrm{A} / \mathrm{V})$} & \multicolumn{2}{|c|}{$2^{\text {nd }}$ order $\left(\mathrm{A} / \mathrm{V}^{2}\right)$} & \multicolumn{2}{|c|}{$3^{\text {rd }}$ order $\left(A / V^{3}\right)$} \\
\hline $\mathrm{g}_{\mathrm{m} 1}^{\mathrm{I}}$ & $0.389 \mathrm{~m}$ & $\mathrm{~g}_{\mathrm{m} 2}^{\mathrm{I}}$ & $0.7 \mathrm{~m}$ & $\mathrm{~g}_{\mathrm{m} 33}^{\mathrm{I}}$ & $-3.83 m$ \\
\hline $\mathrm{g}_{01}^{\mathrm{I}}$ & $6.61 \mathrm{u}$ & $\mathrm{g}_{02}^{\mathrm{I}}$ & 0 & $\mathrm{~g}_{03}^{\mathrm{I}}$ & 0 \\
\hline $\mathrm{g}_{\mathrm{m} 1}^{\mathrm{II}}$ & $2.79 \mathrm{~m}$ & $\mathrm{~g}_{\mathrm{m} 2}^{\mathrm{II}}$ & $9.8 \mathrm{~m}$ & $\mathrm{~g}_{\mathrm{m} 3}^{\mathrm{II}}$ & $65.2 \mathrm{~m}$ \\
\hline $\mathrm{g}^{\mathrm{II}}{ }_{01}$ & $39.0 \mathrm{u}$ & $\mathrm{g}^{\mathrm{II}}{ }_{02}$ & $-1.01 \mathrm{u}$ & $\mathrm{g}_{03}^{\mathrm{II}}$ & $6.18 \mathrm{u}$ \\
\hline & & $\mathrm{g}_{\mathrm{m} 101}^{\mathrm{II}}$ & $14.0 \mathrm{~m}$ & $\mathrm{~g}_{\mathrm{m} 201}^{\mathrm{II}}$ & $6.64 \mathrm{~m}$ \\
\hline & & & & $\mathrm{g}^{\mathrm{II}} \mathrm{m}_{102}$ & $5.21 \mathrm{u}$ \\
\hline
\end{tabular}

The two nonlinear current elements $g_{N L}^{I}\left(V_{1}, V_{2}\right)$ and $g_{N L}^{I I}\left(V_{2}, V_{o}\right)$ model the nonlinearities of the two stages, respectively. Both nonlinear functions are assumed bivariate. The numerically identified nonlinearity coefficients are listed in Table 1.

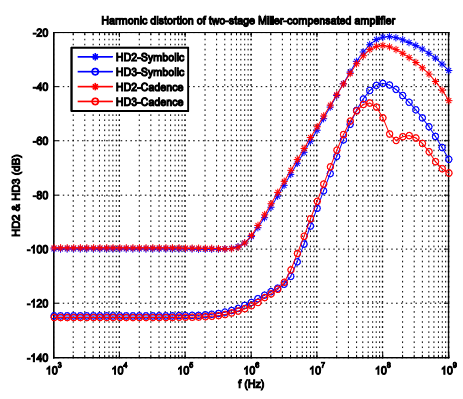

FIGURE VI. HD2 AND HD3 OF THE AMPLIFIER 


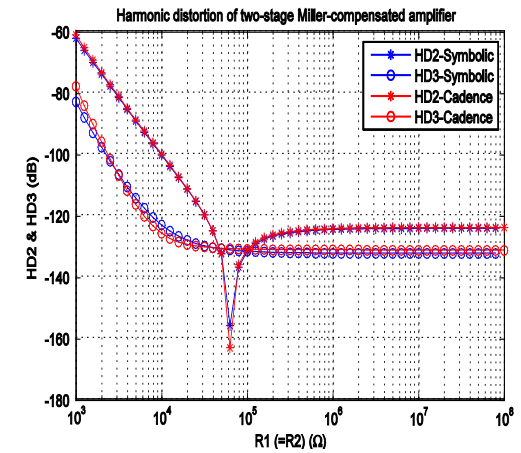

FIGURE VII. SWEEPING RESULTS OF HD2 AND HD3 AT F=1KHZ AGAINST R2 (= R1)

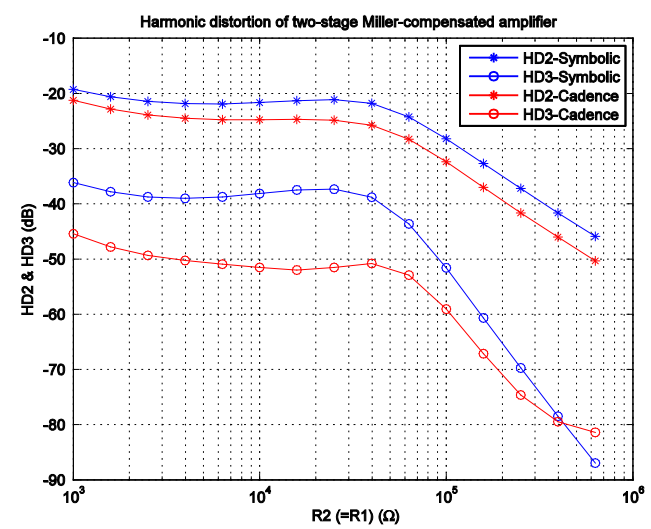

FIGURE VIII. SWEEPING RESULTS OF HD2 AND HD3 NEAR THE PEAK (F = 100MHz) AGAINST R2 (= R1)

In experiment a $100 \mathrm{mVp}-\mathrm{p}$ input signal is applied to the closed-loop circuit. The HD2 and HD3 curves calculated by the symbolic method are drawn in fig. 6 where a comparison to the Cadence simulation result is made. Two sweep results are plotted in fig. 7 and fig. 8, where the former is fixed at the frequency point $f=1 \mathrm{kHz}$ (near dc) and the latter is at GBW. The sweep time was 5.4 seconds by symbolic program and 269.2 seconds by Cadence. We observe from fig. 7 that there exists a value of R2 that makes the dc value of HD2 zero. This phenomenon was discussed in [4] as well. Although the sweep result plotted in fig. 8 has appreciable discrepancy due to inaccuracy of the symbolic method at high frequency, the variation trend is still captured.

\section{CONCLUSION}

A symbolic distortion analysis method has been presented. The method is based on the Volterra series theory and a stagebased nonlinearity modeling strategy. The compact modeling approach greatly simplifies the cost of symbolic analysis. Application to the distortion analysis of an opamp circuit has demonstrated the satisfactory accuracy in the frequency range up to the gain-bandwidth frequency. Significant speedup over Cadence Spectre simulation has been observed.

\section{ACKNOWLEDGEMENT}

This research was supported in part by the Natural Science Foundation of China under Grant No. 61176129 and by a joint research project sponsored by Synopsys, Inc. (2010-2014).

\section{REFERENCES}

[1] P. Wambacq, G. G. E. Gielen, P. R. Kinget, and W. Sansen, "Highfrequency distortion analysis of analog integrated circuits," IEEE Trans. on Circuits and Systems-II: Analog and Digital Signal Processing, vol. 46, no. 3, pp. 335-345, March 1999.

[2] G. Palumbo and S. Pennisi, "High-frequency harmonic distortion in feedback amplifiers: analysis and applications," IEEE Trans. on Circuits and Systems-I: Fundamental Theory and Applications, vol. 50, no. 3, pp. 328-340, March 2003.

[3] S. O. Cannizzaro, G. Palumbo, and S. Pennisi, "Distortion analysis of Miller compensated three-stage amplifiers," IEEE Trans. on Circuits and Systems- I: Regular Papers, vol. 53, no. 5, pp. 961-976, May 2006.

[4] Y. Miao and Y. Zhang, "Distortion modeling of feedback two-stage amplifier compensated with Miller capacitor and nulling resistor," IEEE Trans. on Circuits and Systems-I: Regular Papers, vol. 59, no. 1, pp.93105, January 2012.

[5] L. O. Chua and C. Y. Ng, "Frequency-domain analysis of nonlinear systems: general theory," IEE J. Electronic Circuits and Systems, vol. 3, no. 4, pp. 165-185, July 1979.

[6] P. Wambacq and W. Sansen, Distortion Analysis of Analog Integrated Circuits. Norwell, MA, USA: Kluwer Academic Publishers, 1998.

[7] S. A. Maas and A. Crosmun, "Modeling the gate I/V characteristic of aGaAs MOSFET for Volterra-Series analysis," IEEE Trans. on Microwave Theory and Techniques, vol. 37, no. 7, pp. 1134-1136, July 1989.

[8] G. Shi, "Graph-pair decision diagram construction for topological symbolic circuit analysis," IEEE Trans. on Computer-Aided Design of Integrated Circuits and Systems, vol. 32, no. 2, pp. 275-288, February 2013. 